\title{
ANALYSE DU DISCOURS DE TRAVAILLEURS CONFRONTÉS À DES \\ CHANGEMENTS ORGANISATIONNELS : UNE PERSPECTIVE TRANSACTIONNELLE
}

\author{
$\underline{\text { Titre court : Perception des changements }}$
}

Par F. De Zanet*, I. Hansez**, M. Bossut**, C. Vandenberghe* et V. De Keyser**

\section{SUMMARY}

ANALYSIS OF THE DISCOURSE OF WORKERS CONFRONTED WITH ORGANIZATIONAL CHANGES: A TRANSACTIONAL PERSPECTIVE

Downsizing, restructuring and layoffs have become a recurrent practice among companies today. If consequences of such major changes on individual well-being are well documented, little is known about the nature of work environment changes which workers are confronted with and how they appraise these events. The objective of the present study was to combine quantitative and qualitative data in order to get a better understanding of organizational changes, using a transactional perspective. Fifty-seven workers from seven companies were interviewed. Interviews were transcripted and analyzed along three axes : (a) What were the changes workers were confronted with? (b) How did they react to these changes? and (c) How did they perceive the way their company dealt with these changes? Results indicate that workers were mainly confronted with changes among colleagues, supervisors, in workload as well as changes in task content. Moreover, it appears that not all changes were appraised negatively. Concerning the consequences reported by workers, the findings usually reported in literature were supported by the current data. Workers reported a general deterioration of their quality of life, a degradation of working climate and also more job insecurity associated with 
organizational changes. Finally, workers indicated that the way companies managed changes was not really efficient. Workers complained mainly about information. They reported that they were not or poorly informed about future changes. In conclusion, this study underscores the interest of a qualitative analysis of the experience of workers confronted with organisational changes.

Key words: Organizational Changes, Transactional Perspective, Analysis Of Discourse, WellBeing At Work

*Unité de Psychologie Sociale et des Organisations, Université catholique de Louvain, Place Cardinal Mercier, 10, 1348 LOUVAIN-LA-NEUVE, Belgique. E-mail :

fabrice.dezanet@psp.ucl.ac.be

**Service de Psychologie du Travail, Bat. B-32 FAPSE, Université de Liège, 4000 LIEGE, Belgique. E-mail : ihansez@ulg.ac.be 


\section{INTRODUCTION}

Il est maintenant reconnu combien la globalisation de l'économie a accentué la compétitivité internationale et la pression exercée sur les entreprises (Ladipo \& Wilkinson, 2002). Cette accentuation de la concurrence incite les gestionnaires à compresser au maximum les coûts de fonctionnement, ce qui, pour nombre d'entre eux, se résume souvent à la compression des seuls coûts salariaux. En conséquence, et bien que la course à la rentabilité puisse prendre diverses formes, la stratégie la plus visible, et sans doute la plus répandue, reste la réduction des effectifs (McKinley, Zhao, \& Rust, 2000). Les licenciements collectifs représentent, tant en Europe qu'aux États-Unis, un événement habituel dans l'environnement économique. Ainsi, Shaw et Barret-Power (1997) rapportent que ces restructurations sont souvent récurrentes et qu'elles permettent rarement d'atteindre les objectifs assignés en termes de productivité. Ces raisons expliquent certainement pourquoi la réduction des effectifs est le changement organisationnel majeur le plus souvent évoqué à travers la littérature en psychologie organisationnelle.

Selon Armenakis et Bedeian (1999), les études relatives aux changements organisationnels ont examiné quatre thématiques principales, à savoir le contenu des changements, leur contexte, le processus même du changement et, enfin, leurs conséquences. Les psychologues du travail se sont plus particulièrement intéressés aux conséquences des changements organisationnels, et celles-ci ont été essentiellement envisagées selon trois grandes perspectives. La première, davantage centrée sur l'entreprise, aborde cette problématique de façon globale, s'intéressant aux conséquences des changements majeurs, tels que fusions, acquisitions, restructurations ou délocalisations (Armenakis \& Bedeian, 1999; Kozlowski, Chao, Smith, \& Hedlund, 1993). Tout d'abord, ces changements affectent directement le niveau de stress et de bien-être des travailleurs (Moyle \& Parkes, 1999). On observe également une détérioration de la santé en général (Ferrie, Shipley, Marmot, Stansfeld, \& Smith, 1995 ; Kivimäki, Vahtera, Pentti, \& Ferrie, 2000), une augmentation de la consommation de tabac (Kivimäki et al., 2000), ou encore une augmentation des absences pour raisons médicales (Kivimäki et al., 2000). En outre, ces changements se révèlent tout aussi peu profitables pour les entreprises elles-mêmes. Plusieurs recherches rapportent que ces changements sont associés à une diminution de l'implication organisationnelle (ArmstrongStassen, 1998 ; Kozlowski et al., 1993), à une diminution du rendement au travail et de la productivité de l'entreprise (Armstrong-Stassen, 1998 ; Cascio, 1993 ; Kozlowski et al., 1993), ou encore à une augmentation de l'intention de quitter l'entreprise (Kozlowski et al., 1993). 
Ces études ont eu le mérite de mettre en évidence combien ces changements majeurs peuvent affecter négativement le fonctionnement et les performances d'une entreprise, mais aussi et surtout le bien-être des travailleurs. Elles ont alerté les responsables sur l'indispensable prise en compte des facteurs humains dans la réussite de tels changements et leur ont proposé des conseils en vue de mieux les gérer. En effet, le paradoxe de ces changements majeurs, selon Mishra et Spreitzer (1998), est qu'ils ont pour conséquence d'altérer ce qui est essentiel à leur réussite, à savoir la confiance des travailleurs dans leur entreprise. Toutefois, pour la majorité de ces études, le changement est souvent isolé de son contexte, mesuré ponctuellement et considéré comme la cause principale des conséquences négatives observées. Or, selon Buono et Bowditch (1989), ces changements majeurs sont souvent planifiés sur le long terme et leurs conséquences peuvent s'étaler sur de très longues périodes. Ces auteurs notent ainsi qu'il faut au moins deux années pour pouvoir évaluer l'impact d'une fusion. C'est également ce que souligne Armstrong-Stassen (1997) en faisant remarquer que le fait d'avoir été confronté à un seul licenciement collectif peut produire des dommages difficilement réparables. Négligeant souvent cette dimension temporelle, cette première perspective rend donc peu compte des changements concrets vécus par les travailleurs et des processus psychologiques à l'origine de la variabilité des réactions individuelles.

La seconde perspective, moins développée dans la littérature, est davantage centrée sur les travailleurs. S'inspirant entre autres du courant ethnologique, cette approche envisage plutôt les changements affectant les entreprises sous l'angle de l'évolution des conditions de travail. En effet, pour les travailleurs, la nouvelle donne économique a profondément modifié de nombreux aspects de leur travail, telles que les relations entre employeurs et employés, les conditions de travail ou encore la stabilité de l'emploi. C'est également ce que soulignent Pichault, Warnotte et Wilkin (1998) en indiquant que, même si le management ne modifie pas intentionnellement l'organisation du travail, celle-ci est inévitablement affectée par les macrochangements réalisés à l'échelle de l'entreprise. Pour les travailleurs, cette nécessaire réorganisation du travail se traduit la plupart du temps par des exigences plus fortes en termes de quantité de travail à réaliser, de compétences à développer et à maîtriser ou encore de responsabilités à assumer.

Ainsi, diverses recherches se sont intéressées plus particulièrement à l'intensification du travail (Burchell, 2002 ; Wichert, 2002), aux pratiques de flexibilité (Hudson, 2002) ou encore à l'insécurité d'emploi (Armstrong-Stassen, 1998 ; De Witte, 1999 ; Ferrie et al., 1995 ; Ferrie, Shipley, Marmot, Martikainen, Stansfeld, \& Smith, 2001 ; Kivimäki et al., 2000 ; Kozlowski, et al., 1993; Wichert, 2002). S'inscrivant dans ce climat de changements perpétuels des 
entreprises modernes, ces recherches tentent d'appréhender de façon plus pertinente le caractère temporel et dynamique des nouvelles situations de travail. Cette perspective de recherche accorde au travailleur un rôle actif dans la mesure où celui-ci interprète et donne du sens aux événements auxquels il est confronté. Ce processus de construction de sens va influencer la façon dont les membres d'une organisation vont comprendre et s'adapter aux changements. C'est notamment ce qu'Isabella (1990) a montré dans une étude sur des membres de direction d'une entreprise. Par ailleurs, Bareil et Savoie (1999) évoquent sept phases de préoccupations. Ces phases permettent de décrire le processus de transition à travers lequel les travailleurs assimilent et s'adaptent aux changements organisationnels auxquels ils peuvent être confrontés. Cette perspective permet donc de mieux rendre compte de la complexité des perceptions et des réactions des travailleurs. Elle ouvre aussi la voie à des possibilités d'intervention dont l'objectif est d'agir directement sur les véritables sources du malaise des travailleurs.

Quant à la troisième perspective de recherche, elle a pour objectif de décrire la transaction qui se joue entre le travailleur et son environnement. En effet, l'examen des deux premières perspectives met clairement en évidence combien il est délicat de rendre compte de la variabilité des réactions individuelles. En ne prenant en compte que la seule exposition à un changement majeur, ne risque-t-on pas de masquer les véritables causes et mécanismes à l'origine de la détérioration du bien-être mise en évidence dans nombre d'études ? Afin de mieux comprendre comment les changements organisationnels majeurs affectent les travailleurs, ne faudrait-il pas également tenir compte de la signification attribuée par les travailleurs à ces changements?

La signification accordée aux événements de l'environnement auquel un individu est confronté est au cœur du modèle transactionnel du stress défini initialement par Lazarus et Folkman (1984) (voir aussi Mackay \& Cooper, 1987) et généralisé par la suite aux études sur les changements organisationnels par Mack, Nelson et Quick (1998). Trois éléments sousjacents caractérisent ce type de modèle. Tout d'abord, il s'agit d'un modèle qui conçoit la personne et l'environnement dans une relation dynamique, mutuellement réciproque et bidirectionnelle. Des caractéristiques séparées de la personne et de l'environnement convergent pour donner de nouvelles significations via le processus d'évaluation de la situation. Ensuite, ce modèle reconnaît, de manière implicite, un processus impliquant des changements au fil du temps, en différenciant les conséquences immédiates des conséquences à long terme du stress sur l'adaptation. Enfin, le phénomène de stress est conçu comme un processus cyclique constamment réitéré au fur et à mesure des situations de confrontation rencontrées (De Keyser 
\& Hansez, 1996). Dans cette perspective, Mack et al. (1998) conçoivent le changement comme un stresseur potentiel et l'impact de ce changement comme un phénomène individuel avec une variabilité élevée. Selon Mack et al. (1998), les facteurs individuels et/ou organisationnels, via leur impact sur la perception, expliquent pourquoi les mêmes situations objectives peuvent être vécues de manière extrêmement différente.

La recherche "Flexihealth" vise à établir un lien entre les changements majeurs, la perception des modifications des conditions de travail qu'ils impliquent et le bien-être. Dans le cadre de cette recherche, nous avons adopté cette approche transactionnelle du stress dans un contexte de changement organisationnel. La présente étude a constitué une étape préliminaire de ce projet de recherche et visait à cerner les problématiques rencontrées par les travailleurs dans un contexte de changement organisationnel. Nous basant sur la perspective transactionnelle, trois questions ont orienté l'analyse des données recueillies, à savoir: (1) quels sont les changements auxquels les travailleurs ont été confrontés et comment ces changements ont-ils été évalués ? (2) comment les travailleurs ont-ils réagi à ces changements et quelles en ont été les conséquences ? et (3) comment la gestion des changements par l'entreprise a-t-elle été perçue?

Le recours à une méthodologie qualitative nous semblait tout à fait approprié dans le contexte de cette étude préliminaire. Selon Starrin (1994), cité par Roald et Edgren (2001), une méthodologie qualitative permet, en effet, une analyse plus approfondie de l'expérience par les travailleurs des changements organisationnels auxquels ils ont été confrontés. Notre méthodologie incluait également un volet quantitatif dans la mesure où nous avons examiné de façon systématique les changements auxquels les travailleurs ont été confrontés. Alors que les données qualitatives nous ont permis de mieux comprendre comment les travailleurs ont perçu et ont réagi aux changements, les données quantitatives ont permis une description et une comparaison plus systématiques des situations rencontrées par les travailleurs.

Notre démarche d'analyse des données s'est appuyée sur les volets qualitatif et quantitatif de l'enquête. Nous avons combiné ces deux méthodologies pour répondre aux trois axes de lecture décrits ci-dessus. Lors de la discussion, nous chercherons toutefois à mettre en évidence les apports spécifiques de ces deux méthodologies.

\footnotetext{
${ }^{1}$ Le projet de recherche Flexihealth a été subventionné par les Services Scientifiques, Techniques et Culturels (Belgique) dans le cadre du programme d'appui scientifique à la protection des travailleurs (Phase II, 1999-2003).
} 


\section{1. NATURE ET PERCEPTION DES CHANGEMENTS}

Les recherches sur les changements organisationnels majeurs s'étant le plus souvent focalisées sur la réduction des effectifs, il existe finalement bien peu de données sur les changements rencontrés concrètement par les travailleurs dans leur environnement de travail. Jusqu'à présent, aucune étude n'a véritablement recensé de façon systématique les changements vécus par les travailleurs dans un contexte de changement organisationnel. Notre étude se propose donc, dans un premier temps, de dresser une liste des changements rencontrés par les travailleurs de notre échantillon et d'en évaluer l'occurrence réelle.

En outre, bien que de nombreux auteurs insistent sur l'importance de la perception des changements (Armstrong-Stassen, 1997; Lawson \& Angle, 1998), les aspects cognitifs spécifiques au modèle transactionnel, plus précisément l'évaluation individuelle des changements, sont souvent négligés. Notre étude se propose donc, dans un second temps, d'examiner dans quelle mesure les changements rencontrés ont été jugés comme favorables ou défavorables par les travailleurs. Postulant que les mêmes événements objectifs et les conséquences qu'ils impliquent ne sont pas forcément perçus de façon identique par tous les travailleurs, nous nous attendions à une variabilité importante des évaluations à propos des changements.

\section{2. REPONSES AUX CHANGEMENTS ET CONSEQUENCES}

Bien que cet aspect des changements organisationnels ait été davantage étudié, notre étude se propose de relever les principales conséquences évoquées spontanément par les travailleurs de notre échantillon. A ce titre, notre étude a permis de comparer dans quelle mesure les réactions évoquées spontanément recouvrent les variables utilisées le plus souvent dans les études empiriques.

\section{3. GESTION DES CHANGEMENTS PAR L'ENTREPRISE}

La manière dont les changements sont introduits dans l'entreprise est souvent présentée comme un déterminant majeur de la manière dont les changements seront perçus et vécus par les travailleurs (Callan, Terry, \& Schweitzer, 1994 ; Martin, 1999 ; Mishra \& Spreitzer, 1998 ; Reichers, Wanous, \& Austin, 1997; Schweiger \& Denisi, 1991). On peut toutefois se demander dans quelle mesure il existe un décalage entre les pratiques recommandées par les chercheurs ou intervenants et les pratiques réellement rencontrées par les travailleurs. Notre étude se propose donc d'examiner dans quelle mesure les travailleurs ont eu le sentiment que l'entreprise a géré le changement de façon à favoriser leur bien-être. 


\section{MÉTHODOLOGIE}

\section{1. ÉCHANTILLON}

La procédure suivie en vue de constituer notre échantillon de travailleurs à interviewer s'est déroulée en deux temps. Dans un premier temps, nous avons pris contact avec plusieurs entreprises ayant connu, durant les deux dernières années, un changement organisationnel majeur (ex : licenciement collectif, fusion, réorganisation importante du travail, etc.). Dans un second temps, nous avons déterminé, en accord avec un responsable de la gestion des ressources humaines de l'entreprise, les travailleurs pressentis pour l'interview. Le choix des travailleurs a été établi dans un souci de maximiser la diversité des personnes rencontrées. Nous souhaitions, en effet, rencontrer des travailleurs de statut professionnel, d'âge et d'ancienneté variés et dont on pouvait également penser que l'expérience des changements serait diversifiée. Dans certains cas, nous n'avons toutefois pas eu accès à tous les travailleurs de l'entreprise, mais seulement à un département en particulier. Il était enfin demandé à chaque travailleur s'il était d'accord d'être interviewé.

En définitive, notre échantillon final comportait 7 entreprises ou départements et 57 travailleurs. En effet, pour des raisons de fiabilité des données, seuls 57 des 64 travailleurs initialement interviewés ont été pris en compte dans les analyses. En ce qui concerne les entreprises, celles-ci appartenaient tant au secteur public que privé. Elles étaient actives dans des secteurs aussi variés que la production, le transport, la sécurité ou l'orientation professionnelle. En ce qui concerne les travailleurs, notre échantillon final était composé de 46 hommes $(80,7 \%)$. L'échantillon était composé, à part égale, d'ouvriers et d'employés (Tableau 1). En moyenne, les travailleurs étaient âgés de 41 ans $(\underline{E T}=9,4)$. L'ancienneté moyenne des travailleurs était de 18,5 ans $(\underline{\mathrm{ET}}=10,1)$. Enfin, la majorité des travailleurs, soit 52 personnes (91,2\%), bénéficiait d'un contrat à durée indéterminée.

Tableau 1. Répartition selon la catégorie professionnelle des travailleurs de l'échantillon final Sample distribution according to workers' professional status

\section{2. COLLECTE DES DONNEES}

Les données ont été récoltées par la méthode de l'interview. La durée moyenne d'une interview était, approximativement, d'une heure. Celle-ci était intégralement enregistrée sur cassette audio et comportait deux volets principaux. Le premier volet, qualitatif, consistait en une interview semi-structurée. Le guide pour l'interview comptait 10 questions ouvertes 
(annexe 1). Celles-ci permettaient d'appréhender la nature et le déroulement des changements vécus par la personne ainsi que les conséquences de ces changements tant pour l'entreprise, en général, que pour le travailleur, en particulier.

Le deuxième volet, davantage quantitatif, visait à évaluer de façon plus précise comment les changements majeurs au niveau de l'entreprise s'étaient traduits au niveau de l'environnement de travail proche. En conséquence, nous avons élaboré une liste de changements qui, dans un contexte de changement organisationnel, étaient susceptibles de se produire dans l'environnement de travail proche. Une première liste a été élaborée à partir, d'une part, d'un recueil d'information dans la littérature et, d'autre part, à travers des discussions avec des informateurs-clés. En définitive, nous avons retenu 17 changements relevant de 6 grandes catégories : (1) les changements relevant des aspects formels relatifs au contrat de travail (contrat de travail, horaires de travail et rémunération), (2) les changements relevant des pratiques de management (supérieur hiérarchique direct, contrôle du travail par la hiérarchie, critères et procédures d'évaluation du travail et méthodes de management), (3) les changements relevant de l'enrichissement des tâches (niveau de responsabilité, autonomie dans le travail et compétences nécessaires à l'accomplissement du travail), (4) les changements relevant des tâches à effectuer (nature des tâches, charge de travail et manière de travailler), (5) les changements relevant de l'environnement social de travail (collègues et relation avec les clients), et (6) les changements relevant de l'environnement physique de travail (lieu de travail et aménagement du lieu de travail).

Lors de l'interview, nous demandions au travailleur s'il avait été confronté, durant les deux dernières années, à chacun des changements répertoriés dans la liste. Si la réponse était positive, nous lui demandions également dans quelle mesure ce changement avait contribué à dégrader ou à améliorer ses conditions de travail. Pour ce dernier point, il était demandé à la personne d'indiquer sa réponse à l'aide d'une échelle de type Likert à 4 points allant de 1 (amélioration ou dégradation faible des conditions de travail) à 4 (amélioration ou dégradation importante des conditions de travail). Si la personne indiquait que ce changement n'avait pas amélioré ou dégradé ses conditions de travail ou si elle mentionnait que les bénéfices contrebalançaient les inconvénients, nous attribuions la valeur 0 à la réponse. Par contre, si la personne ne pouvait exprimer d'avis par rapport au caractère favorable ou défavorable du changement, la réponse était codée comme manquante. 


\section{3. ANALYSE DES DONNEES}

En ce qui concerne les données qualitatives, l'analyse s'est déroulée en trois étapes. Dans un premier temps, les enregistrements des interviews ont été intégralement retranscrits. A l'issue de ce processus, seules 57 interviews ont été retenues, 7 ayant été écartées du matériel d'analyse en raison de difficultés techniques liées à la retranscription.

Dans un second temps, le matériel d'analyse a été encodé par le premier auteur à l'aide du logiciel Nvivo (Richards, 1999). Le matériel a été découpé en « unités de sens » et chaque unité a été codée selon une catégorie aussi proche que possible du sens émis par l'interviewé. Durant le codage, les différentes catégories construites au fur et à mesure du processus ont été systématiquement comparées afin d'en dégager les similitudes et différences et d'en circonscrire le contenu précis. Ce premier codage, composé d'un grand nombre de catégories très proches du contenu initial, visait à décrire le plus fidèlement possible le matériel récolté. A l'issue de ce processus, 1129 unités de sens ont été regroupées dans 46 catégories. Ces catégories étaient bipolaires de façon à pouvoir rendre compte du fait qu'un même thème pouvait être évoqué soit négativement, soit positivement. Par exemple, une personne pouvait indiquer que les relations avec son supérieur hiérarchique étaient davantage conflictuelles, alors qu'une autre personne soulignait la qualité du soutien reçu par son supérieur. En ce qui concerne le codage, ces deux opinions ont été attribuées à la même catégorie, car elles font référence au même thème, tout en étant distinguées selon leur valence négative ou positive.

Dans un troisième temps, pour des raisons de clarté de présentation, les catégories ont été organisées selon une structure comprenant 4 thématiques générales, elles-mêmes découpées en sous-thèmes. Une première classification des catégories d'analyse a été proposée par le premier auteur et testée par le second auteur. Ce processus de confrontation a permis de résoudre les divergences de classification. En définitive, une structure composée de 18 catégories bipolaires a été retenue. Les catégories sont décrites en annexe 2. Enfin, estimant que l'occurrence d'un même thème chez une même personne ne reflétait pas nécessairement l'importance de ce thème pour la personne, nous avons pris l'option de ne coder qu'une seule fois le thème, même si le travailleur avait évoqué celui-ci à plusieurs reprises lors de son interview.

En ce qui concerne les données quantitatives, nous avons, dans un premier temps, comptabilisé le nombre de personnes qui avaient été confrontées à chacun des changements repris dans notre liste. Dans un second temps, et pour chacun des changements, nous avons classé les répondants dans trois groupes selon leur score à l'échelle de perception du 
changement: (a) les personnes qui ont perçu le changement comme une dégradation de leurs conditions de travail (score compris entre 2 et 4 pour l'échelle «dégradation»), (b) les personnes qui ont perçu le changement comme une amélioration de leurs conditions de travail (score compris entre 2 et 4 pour l'échelle «amélioration ») et (c) les personnes qui n'ont perçu le changement ni comme une dégradation, ni comme une amélioration de leurs conditions de travail (score de 0 ou score de 1 pour l'échelle « dégradation» ou l'échelle « amélioration»).

Au niveau statistique, le chi-carré a été utilisé, tant au niveau des données qualitatives que quantitatives, pour analyser si la répartition des travailleurs était équivalente selon toutes les modalités de perception des changements. Dans certains cas, et contrairement à ce qui est habituellement recommandé, la valeur attendue pour chacune des catégories d'analyse était inférieure à 5. Toutefois, selon Everitt (1977), l'utilisation de la statistique chi-carré peut être utilisée dans un tel cas. Lorsque cette statistique est significative, les jugements peuvent être considérés comme convergents. Par contre, lorsqu'elle est non significative, elle indique une variabilité interindividuelle dans la perception des changements.

\section{RÉSULTATS}

\section{1. AXE 1 - NATURE DES CHANGEMENTS ET PERCEPTION PAR LES TRAVAILLEURS}

En ce qui concerne les changements rencontrés dans leur environnement de travail, les résultats indiquent que plus de $60 \%$ des travailleurs de notre échantillon ont connu un changement de collègues, un changement de supérieur hiérarchique, des changements dans la charge de travail ou dans le contenu des tâches (Tableau 2). L'analyse des données de l'interview semi-structurée (Tableau 3) va dans le même sens. Des changements relatifs aux relations entre collègues, à la charge de travail et aux relations avec le supérieur et la hiérarchie en général sont cités par 53 à 63\% de l'échantillon. Enfin, le contenu de travail est évoqué par $37 \%$ de l'échantillon. La seule différence se situe au niveau du climat relationnel général qui représente le changement le plus cité dans les données qualitatives, mais qui n'est pas appréhendé en tant que tel dans les données quantitatives. En effet, près de $90 \%$ de notre échantillon évoque spontanément des situations relatives au climat relationnel général.

Si on considère maintenant comment ces changements ont été perçus (Tableau 2), les résultats indiquent que les travailleurs soumis à des changements dans la charge de travail et dans le contenu des tâches perçoivent davantage ceux-ci comme une dégradation de leurs conditions de travail $\left(\chi^{2}(2, \underline{\mathrm{N}}=38)=13, \underline{\mathrm{p}}<.05\right.$ et $\chi^{2}(2, \underline{\mathrm{N}}=34)=7,29, \underline{\mathrm{p}}<.05$, respectivement). Les données qualitatives confirment cette tendance (Tableau 3). On constate, 
en effet, que le climat relationnel général, la charge de travail et le contenu du travail sont davantage évoqués négativement par les travailleurs $\left(\chi^{2}(2, \underline{\mathrm{N}}=50)=16,48, \underline{\mathrm{p}}<.0001 ; \chi^{2}(2, \underline{\mathrm{N}}\right.$ $=33)=39,45, \underline{\mathrm{p}}<.0001$ et $\chi^{2}(2, \underline{\mathrm{N}}=21)=26, \underline{\mathrm{p}}<.0001$, respectivement $)$.

Les données quantitatives mettent par contre en évidence que certains changements sont plutôt perçus comme une amélioration des conditions de travail. Il s'agit de changements dans les compétences requises $\left(\chi^{2}(2, \underline{\mathrm{N}}=26)=9,54, \underline{\mathrm{p}}<.01\right)$, le niveau de responsabilité $\left(\chi^{2}(2, \underline{\mathrm{N}}=\right.$ $30)=12,08, \underline{p}<.01)$, l'autonomie $\left(\chi^{2}(2, \underline{N}=29)=20,14, \underline{p}<.0001\right)$, les méthodes de management $\left(\chi^{2}(2, \underline{\mathrm{N}}=23)=6,87, \underline{\mathrm{p}}<.05\right)$ et le lieu de travail $\left(\chi^{2}(2, \underline{\mathrm{N}}=11)=6,72, \underline{\mathrm{p}}<.05\right)$. Enfin, il apparaît que la variabilité des perceptions est plus importante pour les changements dans les relations avec les collègues et avec le supérieur (Tableau 2) $\left(\chi^{2}(2, \underline{N}=43)=0,04\right.$, $\underline{\text { ns }}$ et $\chi^{2}(2, \underline{\mathrm{N}}=41)=5,46, \underline{\mathrm{ns}}$, respectivement). Ces résultats se confirment également dans l'analyse des données qualitatives (Tableau 3$)\left(\chi^{2}(2, \underline{\mathrm{N}}=36)=0,66\right.$, $\underline{\mathrm{ns}}$ et $\chi^{2}(2, \underline{\mathrm{N}}=30)=0,6$, $\underline{\mathrm{ns}}$, respectivement).

Tableau 2. Nature des changements et perception des travailleurs (données quantitatives)

Nature of changes and workers' appraisal (quantitative data)

Tableau 3. Nature des changements et perception des travailleurs (données qualitatives)

Nature of changes and workers' appraisal (qualitative data)

\section{2. AXE 2 - REPONSES AUX CHANGEMENTS ET CONSEQUENCES}

Pour répondre à notre deuxième objectif, à savoir l'examen des conséquences spontanément évoquées par les travailleurs, nous avons choisi, conformément au modèle transactionnel, de distinguer les réactions à court terme des réponses à plus long terme face aux changements vécus. Le tableau 4 décrit les attitudes des travailleurs par rapport aux changements. On constate que $80 \%$ des travailleurs interviewés parlent de leurs craintes ou espoirs par rapport à l'avenir, en termes d'évolution des conditions de travail et d'emploi. Ensuite, près de 50\% des travailleurs de l'échantillon évoquent leur satisfaction ou insatisfaction par rapport aux changements, leur motivation par rapport au travail et enfin la façon dont ils se sont adaptés à la nouvelle situation. Enfin, une minorité, soit 18\%, fait référence aux attitudes de retrait par rapport à l'entreprise.

En termes de perceptions, on constate une variabilité inter-individuelle importante pour la vision prospective $\left(\chi^{2}(2, \underline{\mathrm{N}}=46)=1,34, \underline{\mathrm{ns}}\right)$. Par contre, les perceptions relatives à la satisfaction, la motivation et l'envie de rester dans l'entreprise sont plutôt évoquées 
négativement (respectivement $\chi^{2}(2, \underline{\mathrm{N}}=30)=11,4, \underline{\mathrm{p}}<.01 ; \chi^{2}(2, \underline{\mathrm{N}}=27)=17,55, \underline{\mathrm{p}}<.0001$; $\left.\chi^{2}(2, \underline{\mathrm{N}}=10)=6,2, \underline{\mathrm{p}}<.05\right)$. Les perceptions par rapport aux stratégies d'adaptation semblent particulièrement tranchées : elles sont soit évoquées négativement, soit positivement $\left(\chi^{2}(2, \underline{\mathrm{N}}=\right.$ 29) $=14,55, \underline{p}<.0001)$.

Tableau 4. Attitudes par rapport aux changements (données qualitatives)

Attitudes towards changes (qualitative data)

Si on examine à présent les réponses à long terme aux changements (Tableau 5), le bien-être semble très souvent évoqué par les travailleurs de l'échantillon (84\%). L'analyse statistique présente une prédominance de réponses négatives aux changements $\left(\chi^{2}(2, \underline{N}=48)=\right.$ 20,37, $\mathrm{p}<.0001)$. Les travailleurs se disent davantage stressés, inquiets ou déstabilisés par les changements. Une minorité de travailleurs mentionne la répercussion des changements sur la santé $(28 \%)$ et l'impact de ceux-ci sur la vie privée et familiale (24\%). A nouveau, il s'agit d'évocations négatives (respectivement $\chi^{2}(2, \underline{N}=16)=26,39, \underline{p}<.0001 ; \chi^{2}(2, \underline{N}=14)=$ $22,43, \underline{p}<.0001)$.

Tableau 5. Réponses à long terme aux changements (données qualitatives)

Long term responses to changes (qualitative data)

\section{3. AXE 3 - PERCEPTION DE LA GESTION DES CHANGEMENTS PAR L'ENTREPRISE}

Face à ces réactions négatives par rapport aux changements, il semble intéressant d'examiner dans quelle mesure les travailleurs ont eu le sentiment que l'entreprise a géré le changement de façon à favoriser leur bien-être (Tableau 6). En termes de fréquence de citation, le sentiment d'avoir été informé apparaît en tête pour près de $70 \%$ de travailleurs et est évoqué négativement par un grand nombre $\left(\chi^{2}(2, \underline{\mathrm{N}}=39)=26,03, \underline{\mathrm{p}}<.0001\right)$. La gestion du changement par l'entreprise et la perception de justice sont citées par approximativement 25\% des travailleurs. La consultation ou négociation et la prise en compte des aspects humains sont citées par une minorité de travailleurs, approximativement $8 \%$. Tous les travailleurs qui font référence à la gestion du changement par l'entreprise, à la justice et à la prise en compte des facteurs humains en parlent de manière négative $\left(\chi^{2}(2, \underline{\mathrm{N}}=15)=30, \underline{\mathrm{p}}<.0001 ; \chi^{2}(2, \underline{\mathrm{N}}=13)=\right.$ $26, \underline{p}<.0001$ et $\chi^{2}(2, \underline{N}=4)=8,02, \underline{p}<.05$, respectivement $)$. 
Tableau 6. Déroulement des changements et perception des travailleurs (données qualitatives)

Change progress and workers' appraisal (qualitative data)

\section{DISCUSSION}

L'étude des changements organisationnels figure en bonne place dans la littérature. En effet, depuis quelques années, le contexte économique a profondément changé et, à l'heure actuelle, c'est davantage le changement que la stabilité qui est la norme des entreprises modernes. De nombreux chercheurs s'inquiètent donc de l'impact de ces changements sur la nature des relations qui se tissent entre un travailleur et l'entreprise qui l'emploie. Or, paradoxalement, on sait peu de choses quant à la nature des changements concrètement rencontrés par les travailleurs dans leur environnement de travail. De la même façon, peu d'études ont examiné de façon approfondie le vécu des travailleurs confrontés à de tels changements. Pourtant, il s'agit là de thématiques dont on ne peut nier l'importance, notamment lorsque l'on s'intéresse aux liens entre les changements organisationnels et le bienêtre au travail.

Plusieurs auteurs soulignent combien il est important de prendre en compte la perception des travailleurs. Le modèle transactionnel du stress, adapté par Mack et al. (1998) à la problématique du changement organisationnel, accorde une importance centrale au processus d'évaluation à travers lequel un individu attribue une signification, notamment en termes de menace pour son bien-être, aux événements auxquels il est confronté (Lazarus \& Folkman, 1984). Selon cette perspective transactionnelle, l'origine des réactions individuelles, caractérisées par une variabilité importante, est à rechercher dans la transaction, plus ou moins problématique, d'un individu avec son environnement.

A travers la présente recherche, notre objectif était de mettre en évidence cette variabilité des réactions individuelles dans un contexte de changement organisationnel. La méthodologie de recherche utilisée était à la fois quantitative et qualitative : 57 travailleurs issus de 7 entreprises et dont l'entreprise avait été confrontée, durant les deux dernières années à un ou plusieurs changements majeurs, ont été interviewés à propos de ceux-ci. Notre grille d'analyse des données comportait trois axes, à savoir (1) quels sont les changements auxquels les travailleurs ont été confrontés et comment ces changements ont-ils été évalués ? (2) comment les travailleurs ont-ils réagi à ces changements et quelles en ont été les conséquences ? et (3) comment la gestion des changements par l'entreprise a-t-elle été perçue ?

En ce qui concerne la nature des changements auxquels les travailleurs ont été confrontés et leur évaluation, deux éléments doivent être mis en évidence. Tout d'abord, tant 
les données qualitatives que quantitatives, montrent que les changements les plus fréquemment vécus par les travailleurs lors de changements organisationnels majeurs, sont le changement de collègues et de supérieur hiérarchique, mais aussi les changements dans la charge de travail ou dans le contenu des tâches. Ensuite, les résultats confirment combien les changements font l'objet de perceptions variées de la part des travailleurs. Si certains d'entre eux sont plutôt perçus négativement, comme l'augmentation de la charge de travail, d'autres changements sont perçus positivement. Tel est le cas notamment des compétences requises pour l'exercice de son métier, du niveau de responsabilité ou encore de la plus grande autonomie au travail. Il nous paraît donc intéressant de souligner que les changements organisationnels ne se traduisent pas uniquement de façon négative pour les travailleurs. Certaines évolutions des conditions travail sont perçues plutôt comme un enrichissement et cela, même si elles peuvent augmenter les exigences liées à la fonction. Par contre, d'autres changements font l'objet de perceptions tant négatives que positives (changements parmi les collègues, changement de supérieur hiérarchique, changements dans la manière de travailler, etc.). Plusieurs travailleurs estiment, par exemple, que les relations avec certains collègues se sont améliorées alors que les relations avec d'autres collègues se sont plutôt dégradées.

Il apparaît donc clairement que la seule prise en compte du type de changement n'est pas suffisante pour rendre compte de sa signification négative ou positive pour les travailleurs. $\mathrm{Au}$ contraire, les travailleurs s'engagent activement dans un processus de construction de sens. Bien que cette étude ne permette pas d'en appréhender l'impact, on est en droit de penser que les perceptions individuelles sont modulées, d'une part, par diverses caractéristiques tant organisationnelles qu'individuelles et, d'autre part, par l'évolution des préoccupations des travailleurs à mesure que le changement se déroule. Les résultats soulignent donc la nécessité, lors de l'étude des changements organisationnels, de prendre en compte tant les événements vécus que leur évaluation par les travailleurs.

L'étude apporte également un éclairage utile concernant les conséquences à court et long terme de ces changements majeurs. Les données, conformément à ce qui est postulé par le modèle transactionnel, semblent plutôt confirmer la pertinence de distinguer successivement les stratégies d'adaptation, les conséquences à court terme et les conséquences à long terme. En ce qui concerne les stratégies d'adaptation, certains travailleurs se disent impuissants face aux situations rencontrées. Leur seule stratégie d'adaptation est plutôt passive et consiste, alors, à accepter le changement et à chercher à s’y adapter («il faut faire avec »). D'autres personnes, par contre, essaient d'adopter une vision plus positive du changement («il faut positiver»), vision qui se traduit, notamment, par un espoir en l'avenir. Parmi ces travailleurs, quelques-uns 
adoptent des stratégies plus actives, comme chercher à modifier leur environnement de travail, chercher des solutions aux problèmes qu'ils peuvent rencontrer, se battre pour obtenir une promotion. Pour certains, ces stratégies d'adaptation ont pour seul objectif d'améliorer leur sort. D'autres, par contre, expriment leur envie de se battre pour leur entreprise.

Concernant les conséquences à court et à long terme, soulignons, tout d'abord, que les données vont dans le sens des études empiriques, à savoir que les changements organisationnels sont plutôt associés à une détérioration de la qualité de vie, tant professionnelle que privée. Les travailleurs, rapportent, lors des interviews, qu'ils ont l'impression que la charge de travail s'est alourdie, en termes de quantité de travail, de rythme et de ressources disponibles. Ils sentent aussi une dégradation dans la définition et la clarté de leurs tâches ou dans l'aide qu'ils peuvent obtenir pour réaliser leur travail. Ils rapportent également qu'ils se sentent moins motivés par leur travail et qu'ils ont davantage envie de quitter l'entreprise qu'auparavant. Il est aussi étonnant de constater combien le climat relationnel général est évoqué négativement. De façon générale, les travailleurs ont le sentiment que l'ambiance de travail s'est progressivement détériorée. Selon eux, celle-ci est davantage marquée par l'individualisme, le carriérisme, le manque de solidarité, d'écoute et de reconnaissance. Certains citent également le chantage à l'emploi. Bien qu'une question de l'interview portait spécifiquement sur l'évolution de l'ambiance de travail, les résultats des analyses laissent apparaître que, sur ce sujet, les avis sont convergents.

Dans le même ordre d'idée, les conséquences à long terme sont également évoquées de façon clairement négatives. A l'instar de la majorité des recherches empiriques, les travailleurs rapportent une détérioration de leur bien-être, de leur vie tant privée que familiale, et de leur santé. Parmi les autres conséquences de ces changements, l'insécurité d'emploi nous semble devoir être mise particulièrement en évidence. D' une part, et contrairement à ce que l'on aurait pu imaginer, leur perception de l'avenir est plutôt nuancée, craintes et espoirs coexistant le plus souvent. Les résultats laissent également apparaître que si les personnes interviewées se préoccupent de la continuité de leur emploi, leurs craintes concernent également l'évolution de leurs conditions de travail. A ce titre, ces résultats confirment la conception de l'insécurité d'emploi de Greenhalgh et Rosenblatt (1984). Selon cette conception, la peur de perdre son emploi n'est pas la seule composante de l'insécurité d'emploi. En effet, pour Greenhalgh et Rosenblatt (1984), la crainte de voir certains aspects de son travail se détériorer et la crainte de perdre certains avantages devraient également être intégrées à ce concept.

Enfin, les travailleurs interviewés ont spontanément fait le lien entre des effets directs des changements (par exemple, leurs attitudes au travail) et des effets plus distants, comme une 
détérioration de leur bien-être. A ce titre, il est tout aussi intéressant de constater que les situations problématiques évoquées par les travailleurs dépassent le contexte professionnel. Ces résultats nous engagent donc à considérer l'impact des changements organisationnels sur le bien-être comme multiples et distillés dans le temps.

Le dernier axe d'analyse faisait référence à la façon dont l'entreprise avait géré ces situations de changement. L'analyse des données révèle que les travailleurs ont perçu de façon clairement négative la façon dont leur entreprise a géré les événements. Le principal reproche est le manque d'information. Certains travailleurs rapportent avoir été surpris par certains changements auxquels ils ne s'attendaient pas du tout. Les données laissent apparaître que la qualité des informations fait aussi défaut. Les employés soulignent combien il leur est difficile d'obtenir une information fiable. Certains commentaires laissent à penser que personne, au sein de leur entreprise, ne savait réellement comment les choses allaient se dérouler. Cette information est sans doute une des conséquences du mode de gestion de nombreuses entreprises. En effet, le centre de décision d'une entreprise ne se trouve pas toujours au sein de l'entreprise et, dès lors, même les gestionnaires se trouvent confrontés à l'incertitude du changement, incertitude qu'ils répercutent auprès de leurs collaborateurs. A ce titre, il faut souligner combien le supérieur hiérarchique direct occupe un rôle crucial dans la gestion du changement. Interlocuteur direct et permanent, les travailleurs, dans un contexte de changement, attendent de lui un support technique et émotionnel et une aide dans la compréhension des événements auxquels ils sont ou vont être confrontés. On peut donc penser que les supérieurs directs sont tout particulièrement exposés lors de ces changements et sans doute serait-il intéressant d'analyser de façon plus spécifique leur vécu face à de tels événements.

D’un point de vue méthodologique, il nous semble opportun de mettre en évidence les apports spécifiques des méthodologies qualitatives et quantitatives. D’une part, cette étude offre des arguments en faveur de ceux qui prônent l'utilisation conjointe de données quantitatives et qualitatives (par exemple, Baker, Israël, \& Schurman, 1996; Steckler, McLeroy, Goodman, Bird, \& McCormick, 1992). Les résultats montrent, en effet, combien données qualitatives et quantitatives s'enrichissent mutuellement. Les méthodes qualitatives génèrent des données riches et détaillées respectant la perspective de l'individu. Les méthodes quantitatives permettent de récolter des données factuelles valides et généralisables. Le parallélisme entre les changements cités de manière spontanée et l'occurrence des changements de la liste pré-établie en est une démonstration claire. D'autre part, il est également important de souligner les divergences entre les données qualitatives et quantitatives. A ce titre, il faut 
rappeler qu'une méthodologie qualitative permet, notamment, de se détacher des catégories habituellement utilisées pour décrire les réactions aux changements organisationnels majeurs (Di Salvo, Lubbers, Rossi, \& Lewis, 1995 ; O’Driscoll \& Cooper, 1996) et, en conséquence, d'accéder plus directement aux représentations des travailleurs quant à la nature de leurs réactions et des conséquences des changements auxquels ils ont été confrontés. Ainsi, les travailleurs ont largement fait référence à la détérioration du climat de travail, thématique qui ne ressort pas des données quantitatives.

Toutefois, nous ne pouvons nier que les réponses récoltées par la méthode de l'interview puissent être tributaires de la subjectivité du chercheur. Alors que la méthodologie quantitative laisse moins de place à l'influence du chercheur, les données issues de la méthode de l'interview sont davantage susceptibles d'être contaminées par les connaissances et impressions de l'interviewer. On est donc en droit de se demander dans quelle mesure la méthodologie qualitative permet effectivement d'accéder à des représentations différentes d'un même phénomène ou s'il s'agit plutôt d'un biais méthodologique. En résumé, les méthodologies qualitatives et quantitatives peuvent générer une compréhension différente des mêmes phénomènes. Elles nous semblent complémentaires et leur combinaison permet d'objectiver les données qualitatives et d'enrichir les données quantitatives.

Bien que cette étude ait permis de mieux comprendre le vécu de travailleurs ayant été confrontés à des changements majeurs au sein de leur entreprise, elle souffre de plusieurs limites. Premièrement, il ne faut pas perdre de vue que le découpage du discours rend difficilement compte de la chronologie des événements et de la structure même du discours de l'interviewé. Il serait probablement utile à l'avenir d'envisager des possibilités de mieux rendre compte des aspects dynamiques du discours. Deuxièmement, seuls les travailleurs encore occupés au sein de l'entreprise ont été interviewés. Or, on sait combien les travailleurs licenciés font également partie des victimes de ces changements majeurs. Par ailleurs, on ne peut évidemment écarter l'hypothèse que la variabilité des perceptions puisse être liée à l'histoire particulière des entreprises au sein desquelles ont été menées les interviews.

Enfin, notre étude se limite à une description du vécu. Par rapport à certaines thématiques, une analyse de l'activité aurait permis d'objectiver des situations de travail plus spécifiques, comme la perception d'une détérioration des conditions de travail dans le cas de changement de tâches, par exemple. Mais une telle analyse ne se justifie que si on a pour objectif de comprendre en quoi le changement de tâches est négatif pour le travailleur. Or, la méthodologie utilisée ne permettait pas de mettre en relation ces différentes variables afin d'analyser les mécanismes à travers lesquels l'effet des changements majeurs s'exprime. 
Toutefois, et comme nous l'avons précisé préalablement, cette étude est la première phase d'une recherche plus globale. En effet, sur base de ces premiers résultats, le réseau de recherche Flexihealth a développé un questionnaire s'inscrivant dans une perspective transactionnelle du stress. Ce questionnaire permet, en effet, d'appréhender tant les changements réellement vécus que leur évaluation. Il prend également en compte diverses caractéristiques organisationnelles et individuelles susceptibles de moduler l'effet des changements et de leur évaluation sur le bien-être au travail et la qualité de vie.

\section{REFERENCES}

Armenakis, A.A., \& Bedeian, A.G. (1999). Organizational change: A review of theory and research in the 1990s. Journal of Management, 25, 293-315.

Armstrong-Stassen, M. (1997). The effect of repeated management downsizing and surplus designation on remaining managers: An exploratory study. Anxiety, Stress and Coping, 10, 377-384.

Armstrong-Stassen, M. (1998). Downsizing the Federal Government: A longitudinal study of managers' reactions. Canadian Journal of Administrative Sciences, 15, 310-321.

Baker, E., Israël, B., \& Schurman, S. (1996). Role of control and support in occupational stress: An integrated model. Social Science and Medicine, 43, 1145-1159.

Bareil, C., \& Savoie, A. (1999). Comprendre et mieux gérer les individus en situation de changement organisationnel. Gestion, 24, 86-94.

Buono, A.F., \& Bowditch, J.L. (1989). The human side of mergers and acquisitions. San Francisco, CA: Jossey-Bass.

Burchell, B. (2002). The prevalence and redistribution of job insecurity and work intensification. In B. Burchell, D. Ladipo, \& F. Wilkinson (Eds.), Job insecurity and work intensification (pp. 39-60). London: Routledge.

Callan, V.J., Terry, D.J., \& Schweitzer, R. (1994). Coping resources, coping strategies and adjustment to organizational-change: Direct or buffering effects? Work \& Stress, 8, 372383.

Cascio, W.F. (1993). Downsizing: what do we know? Academy of Management Executive, 7, 95-104. 
De Keyser, V., \& Hansez, I. (1996). Vers une perspective transactionnelle du stress au travail: Pistes d'évaluations méthodologiques. Cahiers de Médecine du Travail, 33, 133-144.

De Witte, H. (1999). Job insecurity and psychological well-being: Review of the literature and exploration of some unresolved issues. European Journal of Work and Organizational Psychology, 8, 155-177.

Di Salvo, V., Lubbers, C., Rossi, A.M., \& Lewis, J. (1995). Unstructured perceptions of workrelated stress: An exploratory qualitative study. In R. Crandall \& P.L. Perrewé (Eds.), Occupational stress: A handbook (pp. 39-50). Bristol, UK: Taylor \& Francis.

Everitt, B.S. (1977). The analysis of contingency tables. London: Chapman \& Hall.

Ferrie, J.E., Shipley, M.J., Marmot, M.G., Martikainen, P., Stansfeld, S.A., \& Smith, G.D. (2001). Job insecurity in white-collar workers: Toward an explanation of association with health. Journal of Occupational Health Psychology, 6, 26-42.

Ferrie, J.-E., Shipley, M.J., Marmot, M.G., Stansfeld, S., \& Smith, G.D. (1995). Health effects of anticipation of job change and non-employment: Longitudinal data from the Whitehall II study. British Medical Journal, 311, 1264-1269.

Greenhalgh, L., \& Rosenblatt, Z. (1984). Job insecurity: Toward conceptual clarity. Academy of Management Journal, 9, 438-448.

Hudson, M. (2002). Flexibility and the reorganisation of work. In B. Burchell, D. Ladipo, \& F. Wilkinson (Eds.), Job insecurity and work intensification (pp. 39-60). London: Routledge.

Isabella, L.A. (1990). Evolving interpretations as a change unfolds: How managers construe key organizational events. Academy of Management Journal, 1, 7-41.

Kivimäki, M., Vahtera, J., Pentti, J., \& Ferrie, J.-E. (2000). Factors underlying the effect of organisational downsizing on health of employees: Longitudinal cohort study. British Medical Journal, 20, 971-975.

Kozlowski, S.W.J., Chao, G.T., Smith, E.M., \& Hedlund, J. (1993). Organizational downsizing: Strategies, interventions, and research implications. In C.L. Cooper \& I.T. Robertson (Eds.), International Review of Industrial and Organizational Psychology Volume 8 (pp. 263-332). New York: John Wiley \& Sons. 
Lapido, D., \& Wilkinson, F (2002). More pressure, less protection. In B. Burchell, D. Ladipo, \& F. Wilkinson (Eds.), Job insecurity and work intensification (pp. 8-38). London: Routledge.

Lawson, M.B., \& Angle, H.L. (1998). Commitment, satisfaction, and regret after a corporate relocation. Group \& Organization Management, 23, 289-317.

Lazarus, R.S., \& Folkman, S. (1984). Stress, appraisal and coping. New York: Springer.

Mack, D.A., Nelson, D.I., \& Quick, J.C. (1998). The stress of organisational change: A dynamic process model. Applied Psychology : An International Review, 47, 219-232.

Mackay, C., \& Cooper, C.L. (1987). Occupational stress and health : Some current issues. In C.L. Cooper \& R. Robertson (Eds.), International Review of Industrial and Organizational Psychology (pp. 167-199). Chichester, UK: Wiley.

Martin, R. (1999). Adjusting to job relocation: Relocation preparation can reduce relocation stress. Journal of Occupational and Organizational Psychology, 72, 231-235.

McKinley, W., Zhao, J., \& Rust, K.G. (2000). A sociocognitive interpretation of organizational downsizing. Academy of Management Review, 25, 227-243.

Mishra, A.K., \& Spreitzer, G.M. (1998). Explaining how survivors respond to downsizing: The roles of trust, empowerment, justice, and work redesign. Academy of Management Review, 23, 567-588.

Moyle, P.J., \& Parkes, K. (1999). The effects of transition stress: A relocation study. Journal of Organizational Behavior, 20, 625-646.

O’Driscoll, M.P., \& Cooper, C.L. (1996). A critical incident analysis of stress-coping behaviours at work. Stress Medicine, 12, 123-128.

Pichault, F., Warnotte, G., \& Wilkin, L. (1998). La fonction ressources humaines face aux restructurations: Trois cas de downsizing. Paris: L'Harmattan.

Reichers, A.E., Wanous, J.P., \& Austin, J.T. (1997). Understanding and managing cynicism about organizational change. Academy of Management Executive, 11, 48-59.

Richards, L. (1999). Using NVivo in Qualitative Research. London: Sage Publications.

Roald, J., \& Edgren, L. (2001). Employee experience of structural change in two Norwegian hospitals. International Journal of Health Planning Management, 16, 311-324. 
Schweiger, D., \& Denisi, A. (1991). Communication with employees following a merger: A longitudinal field experiment. Academy of Management Journal, 34, 110-135.

Shaw, J.B., \& Barrett-Power, E. (1997) A conceptual framework for assessing organization, work group, and individual effectiveness during and after downsizing. Human relations, 50, 109-127.

Starrin, B. (1994). Grounded theory - En metod for kvalitativ analys. In P.-G. Svensson \& B. Starrin (Eds.), Kvalitativa Studier i Praktik och Teori. Studentlitteratur: Lund.

Steckler, A., McLeroy, K.R., Goodman, R.M., Bird, S.T., \& McCormick, L. (1992). Towards integrating qualitative and quantitative methods: An introduction. Health Education Quarterly, 19, 1-8.

Wichert, I. (2002). Job insecurity and work intensification: The effects on health and wellbeing. In B. Burchell, D. Ladipo, \& F. Wilkinson (Eds.), Job insecurity and work intensification (pp. 39-60). London: Routledge. 


\section{Annexe 1 : Guide d'interview}

1. Pouvez-vous nous décrire quels sont les changements les plus significatifs intervenus dans l'entreprise ces deux dernières années?

2. Comment vous ont été communiqués ces changements ?

3. Comment décririez-vous le climat, l'ambiance de travail durant ces deux dernières années (au niveau de l'entreprise, de l'unité, etc.) ?

4. Y a-t-il eu une différence dans le climat de travail de l'entreprise avant et après le changement majeur?

5. Y a-t-il, pour vous, un événement précis qui a fait évoluer le climat de l'entreprise ?

6. Comment avez-vous réagi face aux changements ?

7. Qu'est-ce qui vous a aidé à gérer la transition (personnes, formation, compensation, etc.) ?

8. Les changements majeurs survenus dans votre entreprise ont-ils eu des conséquences en termes de stress? Pouvez-vous expliquer?

9. Comment entrevoyez-vous votre avenir ? (emploi, entreprise, fonction, etc.)

10. Avez-vous quelque chose à ajouter à propos des changements vécus dans votre entreprise et dont nous n'aurions pas encore parlé ? 


\section{CHANGEMENTS VECUS PAR LES TRAVAILLEURS}

\section{Relations avec les collègues}

Il s'agit de la qualité ou du type de relations vécues avec les collègues, du soutien perçu par les collègues.

\section{Relations avec le supérieur et la hiérarchie en général}

Il s'agit de la qualité ou du type de relations vécues avec le supérieur hiérarchique direct ou avec la hiérarchie en général, du soutien perçu par la hiérarchie, de la confiance dans la hiérarchie.

\section{Climat relationnel général}

Il s'agit de la qualité de l'ambiance de travail et/ou de son évolution (amélioration/dégradation). Elle peut se traduire tant en termes de solidarité, de reconnaissance, de considération, d'écoute qu'en termes de carriérisme, d'opportunisme ou de chantage à l'emploi.

\section{Charge de travail}

Cette catégorie réfère à la quantité de travail, la pression liée aux urgences et au contrôle, la répartition des tâches, le rythme de travail, la gestion des absences, les ressources disponibles en personnel et/ou autres moyens.

\section{Contenu du travail}

Cet aspect fait référence non seulement à la définition des tâches, aux missions, mais aussi à la capacité à mener à bien ces tâches ou ces missions, à l'aide possible pour atteindre les objectifs, au sentiment de compétence personnelle, à la formation nécessaire.

\section{ATTITUDES PAR RAPPORT AUX CHANGEMENTS}

\section{Stratégies d'adaptation}

Il s'agit de l'attitude générale du travailleur et son adaptation envers les changements. Il peut soit avoir une attitude active, se prendre en main, faire des choix, soit accepter passivement les changements, être résigné, subir les changements.

\section{Satisfaction par rapport aux changements}

Si le travailleur a une attitude positive, il estime que les changements ont amené des améliorations dans sa situation de travail, il se dit satisfait par les changements. Dans le cas contraire, le travailleur manifeste de la déception, de l'amertume suite aux changements avec une impression de dégradation de la situation.

\section{Motivation par rapport à la nouvelle situation de travail}

Soit le travailleur se dit intéressé par son travail, soit il fait état d'un manque de motivation et d'envie par rapport au travail (lassitude, monotonie, manque de plaisir, découragement, etc.).

\section{Envie de rester au sein de l'entreprise}

Soit le travailleur a envie de rester dans l'entreprise, d'y évoluer et de progresser; soit il est pressé de quitter l'entreprise, d'arriver en fin de carrière, etc. 


\section{Vision prospective}

Le travailleur décrit son avenir avec sérénité et optimisme ou, au contraire, il l'envisage comme incertain et potentiellement délicat.

\section{REPONSES AUX CHANGEMENTS}

\section{Bien-être}

Le travailleur fait mention de calme, de sérénité ou, au contraire, de stress, de tracas, d'inquiétudes, de soucis. Il peut être ou non déstabilisé par les changements.

\section{Vie privée et familiale}

Il s'agit de la conciliation vie professionnelle - vie privée/familiale. Le travailleur peut aussi faire référence au soutien qu'il peut recevoir de la part de sa famille.

\section{Santé}

Cette catégorie décrit dans quelle mesure le travail peut avoir un impact, positif ou négatif, sur la santé.

\section{DEROULEMENT DU CHANGEMENT}

\section{Information}

Le travailleur a l'impression d'être informé ou, au contraire, d'être mis devant le fait accompli sans être tenu au courant. Il peut également percevoir une rétention de l'information à son égard.

\section{Consultation, négociation}

Il s'agit du sentiment d'être consulté pour négocier les changements. Le travailleur peut inversement avoir l'impression que les changements lui sont imposés.

\section{Prise en compte des aspects humains}

Le travailleur a le sentiment que l'entreprise agit avec délicatesse, diplomatie ou, au contraire, qu'elle fait preuve de beaucoup de maladresse et que la communication passe mal.

\section{Gestion du changement par l'entreprise}

Il s'agit de la manière dont l'entreprise a géré les changements en termes d'aménagement des conditions de travail et de conception et de planification des changements.

\section{Justice}

Il s'agit de la manière dont le travailleur peut se sentir favorisé ou défavorisé par rapport à d'autres travailleurs. 


\section{Tableau 1}

$\underline{\text { Répartition selon la catégorie professionnelle des travailleurs de l'échantillon final }}$

Fonction

\begin{tabular}{lccc} 
Entreprise & Ouvrier & Employé & Total \\
\hline Orientation professionnelle & 0 & 2 & 2 \\
Production - 1 & 9 & 5 & 14 \\
Production - & 17 & 7 & 24 \\
Sécurité - 1 & 0 & 2 & 2 \\
Sécurité - 2 & 0 & 3 & 3 \\
Transport - 1 & 0 & 1 & 1 \\
Transport - 2 & 0 & 11 & 11 \\
\hline
\end{tabular}

Total

26

31

57 


\section{Tableau 2}

$\underline{\text { Nature des changements et perception des travailleurs (données quantitatives) }}$

\begin{tabular}{|c|c|c|c|c|c|c|c|}
\hline & & & & Perçu ni comme u. & & & \\
\hline & Nombre de travailleurs & Perçu comme & Perçu comme & amélioration, ni & & & \\
\hline & ayant connu le & une & une & comme une & & & \\
\hline Changements & changement & détérioration & amélioration & dégradation & $\chi^{2}$ & $\underline{\mathrm{d} l}$ & $\mathrm{p}$ \\
\hline Collègues & $51(89.47 \%)$ & $14(32.6 \%)$ & $15(34.9 \%)$ & $14(32.6 \%)$ & 0.04 & 2 & 0.977 \\
\hline Supérieur hiérarchique & $44(77.19 \%)$ & $15(36.6 \%)$ & $19(46.3 \%)$ & $7(17.1 \%)$ & 5.46 & 2 & 0.065 \\
\hline Charge de travail & $41 \quad(71.93 \%)$ & $21 \quad(55.3 \%)$ & $14(36.8 \%)$ & $3(7.9 \%)$ & 13 & 2 & 0.002 \\
\hline Tâches & $36(63.16 \%)$ & $16(47.1 \%)$ & $14(41.2 \%)$ & $4(11.8 \%)$ & 7.29 & 2 & 0.026 \\
\hline Horaires & $32(56.14 \%)$ & $16(51.6 \%)$ & $8(25.8 \%)$ & $7(22.6 \%)$ & 4.71 & 2 & 0.095 \\
\hline Manière de travailler & $32(56.14 \%)$ & $14(46.7 \%)$ & $14(46.7 \%)$ & $2(6.7 \%)$ & 9.6 & 2 & 0.008 \\
\hline Compétences & $31(54.39 \%)$ & $6(23.1 \%)$ & $16(61.5 \%)$ & $4(15.4 \%)$ & 9.54 & 2 & 0.008 \\
\hline Contrôle par la hiérarchie & $30(52.63 \%)$ & $11(39.3 \%)$ & $11(39.3 \%)$ & $6(21.4 \%)$ & 1.78 & 2 & 0.409 \\
\hline Niveau de responsabilité & $30(52.63 \%)$ & $10(33.3 \%)$ & $18(60.0 \%)$ & $2(6.7 \%)$ & 12.8 & 2 & 0.002 \\
\hline Autonomie & $30(52.63 \%)$ & $5(17.2 \%)$ & $21(72.4 \%)$ & $3(10.3 \%)$ & 20.14 & 2 & 0.000 \\
\hline Rémunération & $28(49.12 \%)$ & $8(28.6 \%)$ & $11(39.3 \%)$ & $9(32.1 \%)$ & 0.5 & 2 & 0.779 \\
\hline Méthodes de management & $27(47.37 \%)$ & $9(39.1 \%)$ & $12(52.2 \%)$ & $2(8.7 \%)$ & 6.87 & 2 & 0.032 \\
\hline Environnement de travail & $27(47.37 \%)$ & $6(26.1 \%)$ & $11(47.8 \%)$ & $6(26.1 \%)$ & 2.17 & 2 & 0.337 \\
\hline Relation client & $22(38.6 \%)$ & $8(36.4 \%)$ & $8(36.4 \%)$ & $6(27.3 \%)$ & 0.36 & 2 & 0.834 \\
\hline Contrat de travail & $16(28.07 \%)$ & $2(16.7 \%)$ & $6(50.0 \%)$ & $4(33.3 \%)$ & 2 & 2 & $0.368^{\mathrm{a}}$ \\
\hline Lieu de travail & $14(24.56 \%)$ & $4(36.4 \%)$ & $7(63.6 \%)$ & $0(0.0 \%)$ & 6.72 & 2 & 0.034 \\
\hline Evaluation & $13(22.81 \%)$ & $2(18.2 \%)$ & $7(63.6 \%)$ & $2(18.2 \%)$ & 4.54 & 2 & $0.103^{\mathrm{a}}$ \\
\hline
\end{tabular}

Note. Le pourcentage entre parenthèses correspond à la proportion de travailleurs ayant connu le changement par rapport aux

57 travailleurs de l'échantillon. Les pourcentages mentionnés dans la partie droite du tableau font référence à la répartition des travailleurs ayant évalué le changement comme une amélioration, une détérioration ou encore 'ni amélioration, ni dégradation' des conditions de travail.

${ }^{a}$ La fréquence attendue est inférieure à 5 dans les trois cellules. 


\section{Tableau 3}

\section{$\underline{\text { Nature des changements et perception des travailleurs (données qualitatives) }}$}

\begin{tabular}{|c|c|c|c|c|c|c|c|}
\hline & Nombre de & & Thème & Thème évoqué & & & \\
\hline & travailleurs ayant & Thème évoqué & évoqué & négativement et & & & \\
\hline Catégories & évoqué ce thème & négativement & positivement & positivement & $\chi^{2}$ & $\underline{\mathrm{dl}}$ & $\mathrm{p}$ \\
\hline Climat relationnel général & $50(87.7 \%)$ & 30 & 8 & 12 & 16.48 & 2 & 0.000 \\
\hline & & $60.0 \%$ & $16.0 \%$ & $24.0 \%$ & & & \\
\hline Relations avec les collègues & $36(63.2 \%)$ & 14 & 12 & 10 & 0.66 & 2 & 0.717 \\
\hline & & $38.9 \%$ & $33.3 \%$ & $27.8 \%$ & & & \\
\hline Charge de travail & $33(57.9 \%)$ & 28 & 3 & 2 & 39.45 & 2 & 0.000 \\
\hline & & $84.8 \%$ & $9.1 \%$ & $6.1 \%$ & & & \\
\hline Relations avec le supérieur et la & $30(52.6 \%)$ & 12 & 9 & 9 & 0.6 & 2 & 0.741 \\
\hline hiérarchie en général & & $40.0 \%$ & $30.0 \%$ & $30.0 \%$ & & & \\
\hline Contenu du travail & $21(36.8 \%)$ & 18 & 1 & 2 & 26 & 2 & 0.000 \\
\hline & & $85.7 \%$ & $4.8 \%$ & $9.5 \%$ & & & \\
\hline
\end{tabular}

Note. Le pourcentage entre parenthèses correspond à la proportion de travailleurs ayant évoqué le thème par rapport aux 57 travailleurs de l'échantillon. Les pourcentages mentionnés dans la partie droite du tableau font référence à la répartition des travailleurs ayant évoqué ce thème négativement, positivement ou de façon neutre. 


\section{Tableau 4}

\section{$\underline{\text { Attitudes par rapport aux changements (données qualitatives) }}$}

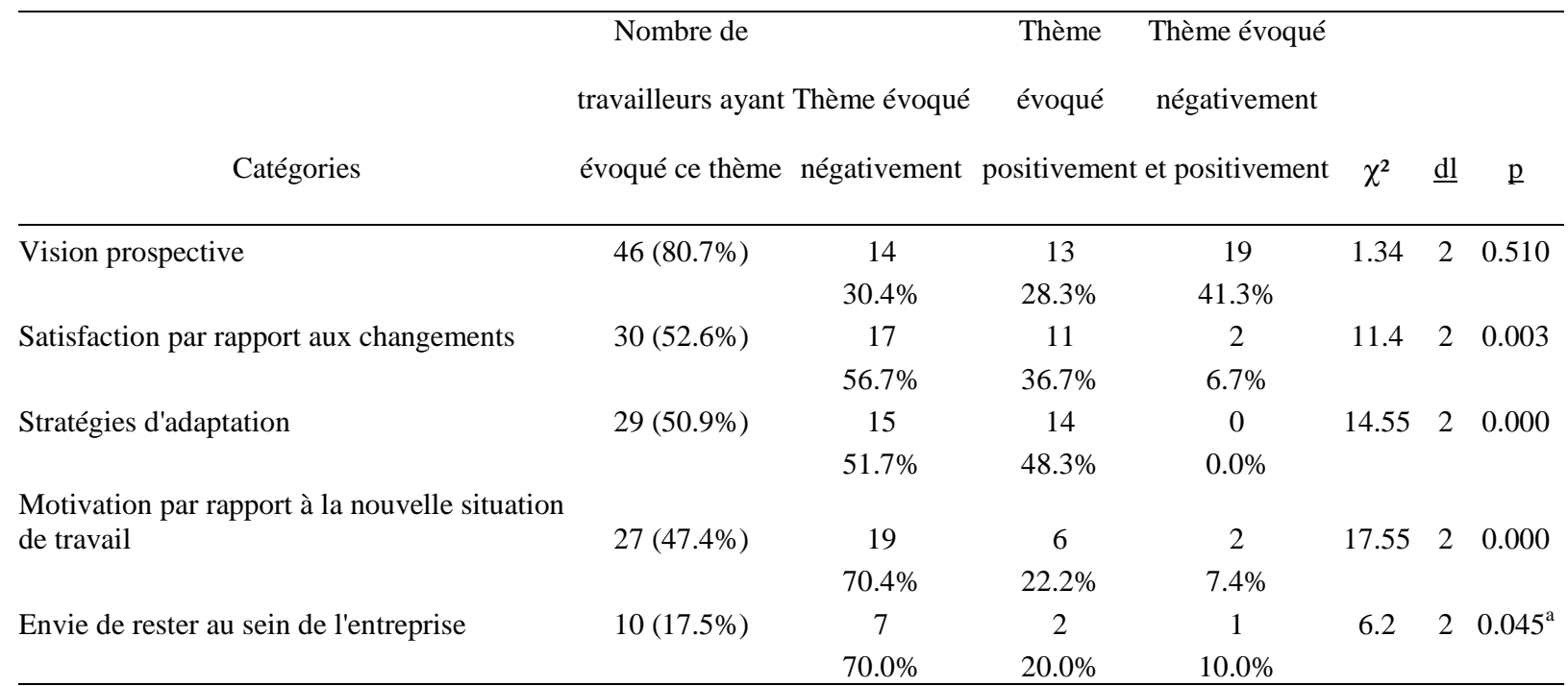

Note. Le pourcentage entre parenthèses correspond à la proportion de travailleurs ayant évoqué le thème par rapport aux 57 travailleurs de l'échantillon. Les pourcentages mentionnés dans la partie droite du tableau font référence à la répartition des travailleurs ayant évoqué ce thème négativement, positivement ou de façon neutre.

${ }^{\text {a }}$ La fréquence attendue est inférieure à 5 dans les trois cellules. 
Tableau 5

$\underline{\text { Réponses à long terme aux changements (données qualitatives) }}$

\begin{tabular}{|c|c|c|c|c|c|c|c|}
\hline \multirow[b]{3}{*}{ Catégories } & Nombre de & & Thème & Thème évoqué & \multirow[b]{3}{*}{$\chi^{2}$} & \multirow[b]{3}{*}{$\underline{\mathrm{dl}}$} & \multirow[b]{3}{*}{$\mathrm{p}$} \\
\hline & travailleurs ayant & Thème évoqué & évoqué & négativement et & & & \\
\hline & évoqué ce thème & négativement & positivement & positivement & & & \\
\hline \multirow[t]{2}{*}{ Bien-être } & $48(84.2 \%)$ & 27 & 2 & 19 & 20.37 & 2 & 0.000 \\
\hline & & $56.3 \%$ & $4.2 \%$ & $39.6 \%$ & & & \\
\hline \multirow[t]{2}{*}{ Santé } & $16(28.1 \%)$ & 15 & 1 & 0 & 26.39 & 2 & 0.000 \\
\hline & & $93.8 \%$ & $6.3 \%$ & $0.0 \%$ & & & \\
\hline \multirow[t]{2}{*}{ Vie privée et familiale } & $14(24.6 \%)$ & 13 & 1 & 0 & 22.43 & 2 & 0.000 \\
\hline & & $92.9 \%$ & $7.1 \%$ & $0.0 \%$ & & & \\
\hline
\end{tabular}

Note. Le pourcentage entre parenthèses correspond à la proportion de travailleurs ayant évoqué le thème par rapport aux 57 travailleurs de

l'échantillon. Les pourcentages mentionnés dans la partie droite du tableau font référence à la répartition des travailleurs ayant évoqué ce thème négativement, positivement ou de façon neutre. 
Tableau 6

$\underline{\text { Déroulement des changements et perception des travailleurs (données qualitatives) }}$

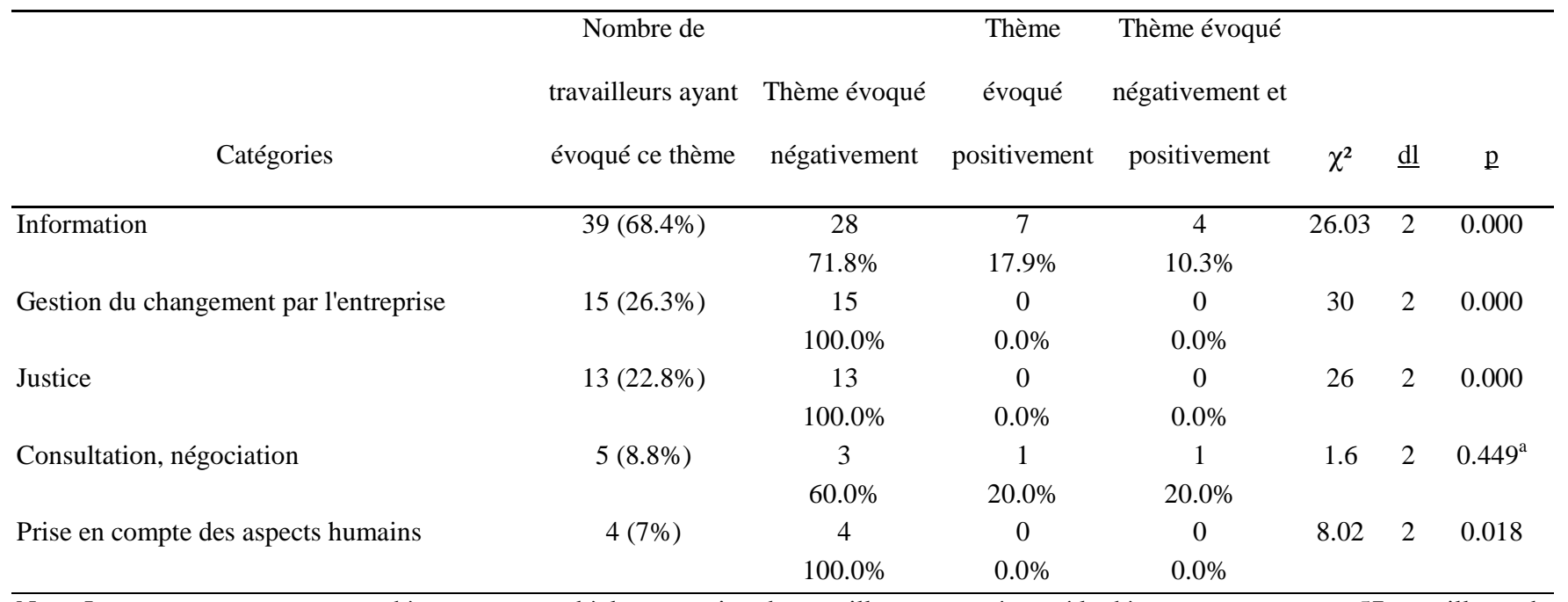

Note. Le pourcentage entre parenthèses correspond à la proportion de travailleurs ayant évoqué le thème par rapport aux 57 travailleurs de

l'échantillon. Les pourcentages mentionnés dans la partie droite du tableau font référence à la répartition des travailleurs ayant évoqué ce thème négativement, positivement ou de façon neutre.

${ }^{\mathrm{a}} \mathrm{La}$ fréquence attendue est inférieure à 5 dans les trois cellules. 


\section{Résumé :}

L'objectif de cette étude était d'analyser la nature des changements concrets rencontrés par des travailleurs dans un contexte de changement organisationnel. La méthodologie utilisée combine des données qualitatives et quantitatives. Cinquante-sept travailleurs ont été interviewés et le matériel récolté a été analysé dans une perspective transactionnelle soulignant l'importance des perceptions individuelles. Les résultats mettent en évidence les changements les plus souvent rencontrés et soulignent la diversité des perceptions quant à ces changements. L'insatisfaction des travailleurs par rapport à la façon dont les changements ont été gérés par l'entreprise ressort également des résultats. Enfin, les données confirment combien ces changements peuvent contribuer à la dégradation du bien-être tant au travail que dans la vie privée.

Mots-clés : Changements organisationnels, Perspective transactionnelle, Analyse du discours, Bien-être au travail. 